\title{
Finite-to-one maps and dimension
}

\author{
by
}

\author{
Jerzy Krzempek (Gliwice)
}

\begin{abstract}
It is shown that for every at most $k$-to-one closed continuous map $f$ from a non-empty $n$-dimensional metric space $X$, there exists a closed continuous map $g$ from a zero-dimensional metric space onto $X$ such that the composition $f \circ g$ is an at most $(n+k)$-to-one map. This implies that $f$ is a composition of $n+k-1$ simple (= at most two-to-one) closed continuous maps. Stronger conclusions are obtained for maps from Anderson-Choquet spaces and ones that satisfy W. Hurewicz's condition $(\alpha)$. The main tool is a certain extension of the Lebesgue-Čech dimension to finite-to-one closed continuous maps.
\end{abstract}

This paper deals with a composition problem, which originates in papers of K. Borsuk, R. Molski, and K. Sieklucki [6, 25]. In [15] the present author proved that every at most $k$-to-one closed map from an $n$-dimensional space is a composition of $(n+1) k-1$ simple (= at most two-to-one) closed maps. Herein, we improve the bound $(n+1) k-1$ to $n+k-1$ without other assumptions added, and to $n+k-2$ if either the domain $X$ of the map to be factored contains no pair of disjoint homeomorphic $n$-dimensional closed subspaces, or the map is irreducible and $X$ satisfies the following condition of W. Hurewicz [12]:

$(\alpha)$ every nowhere dense subspace of $X$ has dimension less than that of $X$.

Our main tool is the use of the concepts of covering and partition dimensions of a map, which were defined in [16] and extend the notion of the Lebesgue-Čech dimension of a space to closed maps. We prove two formulas for calculating the covering dimension cdim of a map and we use them to show that every at most $k$-to-one closed map $f$ from an $n$-dimensional space has $\operatorname{cdim} f \leq n+k-1$. (The statement emphasized in the abstract is, in fact, equivalent to this inequality.) Our formulas for cdim imply well-known theorems on dimension-raising maps by Hurewicz [11, 12], K. Morita [21],

2000 Mathematics Subject Classification: Primary 54F45; Secondary 54C10, 54E40.

Key words and phrases: covering dimension of maps, closed map, at most $k$-to-one map ( = map of order $\leq k)$, composition, theorem on dimension-raising maps, Hurewicz's condition $(\alpha)$, Anderson-Choquet space, Cook continuum. 
J. E. Keesling [13], and A. V. Zarelua [29, 30]. The paper ends with some remarks about Anderson-Choquet and Cook type continua.

Throughout the paper, all spaces are assumed to be metrizable and all maps to be continuous.

1. The covering dimension of closed maps: basic facts. Let $X$ be a topological space and $\mathcal{A}=\left(A_{s}\right)_{s \in S}$ be an indexed collection of subsets of $X$. It is important that the sets $A_{s}$ may be equal for distinct indices $s$. We denote the boundary of $A_{s}$ by $\partial A_{s}$. Given a function $f$ on $X$, we denote the collections $\left(\bar{A}_{s}\right)_{s \in S},\left(\partial A_{s}\right)_{s \in S}$ and $\left(f\left(A_{s}\right)\right)_{s \in S}$ by $\overline{\mathcal{A}}, \partial \mathcal{A}$ and $f(\mathcal{A})$, respectively. All coverings and refinements will be considered as indexed collections of sets, and from now on we omit the word "indexed".

By $|S| \in \mathbb{N} \cup\{\infty\}$ we denote the number of elements in $S$. For any $x \in X$ we define $\operatorname{ord}_{x} \mathcal{A}=\left|\left\{s \in S: x \in A_{s}\right\}\right|$ and $\operatorname{ord} \mathcal{A}=\sup _{x \in X} \operatorname{ord}_{x} \mathcal{A}$. If $X$ is empty, then ord $\mathcal{A}=0$.

1.1. Definition ([16]). Let $f$ be a closed map from a space $X$. The covering dimension cdim $f$ is the least integer $n$ such that every finite open cover of $X$ has a finite closed refinement $\mathcal{F}$ with ord $f(\mathcal{F}) \leq n+1$. If such $n$ 's do not exist, we write $\operatorname{cdim} f=\infty$.

Images of distinct elements of $\mathcal{F}$ may be equal, and then such an image is counted two or more times among the elements of the collection $f(\mathcal{F})$.

It is easily seen that $\operatorname{dim} X \leq \operatorname{cdim} f$ and $\operatorname{dim} f(X) \leq \operatorname{cdim} f$. Since $\operatorname{dim} X=\operatorname{cdimid}_{X}$ for every space $X$, cdim may be viewed as an extension of the Lebesgue-Cech dimension. Moreover, certain properties of dimension extend to maps: the countable and locally finite sum theorems, the completion theorem, and the enlargement theorem - see [16]. The importance of cdim to the study of finite-to-one maps comes from the following

1.2. TheOREM. If $f$ is a closed map from a non-empty space $X$, then statements (a) and (b) below are equivalent, and they imply (c).

(a) $\operatorname{cdim} f \leq n$.

(b) There exists a closed map $g$ from a zero-dimensional space onto $X$ such that the composition $f \circ g$ is at most $(n+1)$-to-one.

(c) $f$ is a composition of $n$ simple closed maps.

The equivalence $(\mathrm{a}) \Leftrightarrow(\mathrm{b})$ is proved in [16, Theorem 6.1]. It extends the well-known Hurewicz-Morita theorem (Morita [21], see also R. Engelking [9, Theorem 4.3.15]), which corresponds to the case $f=\operatorname{id}_{X}$. The implication $(\mathrm{b}) \Rightarrow(\mathrm{c})$ is Theorem 2.2 of [15].

Finally, note that for every finite-to-one map $f$ from a space $X$, we have $\operatorname{dim} X \leq \operatorname{dim} f(X)$ - by the theorem on dimension-lowering maps, see [9, Theorem 4.3.4]. 
2. The first formula for $\operatorname{cdim} f$. Let $f$ be a function into a set $Y$. We define the order of $f$ by ord $f=\sup _{y \in Y}\left|f^{-1}(y)\right|$, and for $k \in \mathbb{N}$ we let

$$
C_{k}(f)=\left\{y \in Y:\left|f^{-1}(y)\right| \geq k\right\}, \quad E_{k}(f)=f^{-1}\left(C_{k}(f)\right) .
$$

If $f$ is a closed map, then $C_{k}(f)$ and $E_{k}(f)$ are $F_{\sigma}$-sets; cf. [9, Lemma 4.3.5], see also our Lemma 2.4(a).

Here we prove the following

2.1. THEOREM. If $f$ is a closed map from a non-empty space and ord $f$ $<\infty$, then

$$
\operatorname{cdim} f=\max \left\{\operatorname{dim} C_{j}(f)+j-1: j=1, \ldots, \text { ord } f\right\} .
$$

The formula above enables easy calculation of the covering dimension cdim. For example, the original Peano map from $[0,1]$ onto $[0,1]^{2}$ has order 4 , and its covering dimension turns out to be 3 . Another example: if $f$ is an exactly $k$-to-one closed map from an $n$-dimensional space, then $\operatorname{cdim} f$ turns out to be $n+k-1$ (to see this use also Lemma 3.2 below). More essential applications will be given in Section 3 .

Before the proof of Theorem 2.1, we need some preparation.

Suppose that $\mathcal{V}=\left(V_{i}\right)_{i=1}^{m}$ is a finite open cover of a space $X$. By the reduction of $\mathcal{V}$ we understand the closed cover, denoted by $[\mathcal{V}]$, that consists of sets $\left[V_{1}\right], \ldots,\left[V_{m}\right]$ defined as follows:

$$
\begin{aligned}
& {\left[V_{1}\right]=\bar{V}_{1},} \\
& {\left[V_{i}\right]=\bar{V}_{i} \backslash\left(V_{1} \cup \cdots \cup V_{i-1}\right) \quad \text { for } i=2, \ldots, m ;}
\end{aligned}
$$

cf. J. M. Aarts, R. J. Fokkink and H. Vermeer [1, Definition 12 and Lemma 13] and S. A. Bogatyı [5, Lemma 3].

Observe that for each $s \geq 2$ and each sequence $1 \leq i_{1}<\cdots<i_{s} \leq m$, we have

$$
\left[V_{i_{1}}\right] \cap \cdots \cap\left[V_{i_{s}}\right] \subset \partial V_{i_{1}} \cap \cdots \cap \partial V_{i_{s-1}} .
$$

Indeed, if $x \in\left[V_{i_{1}}\right] \cap \cdots \cap\left[V_{i_{s}}\right]$, then $x \in \bar{V}_{i_{t}}$ for $t \leq s$. However, $x \notin V_{i_{t}}$ for $t<s$ by the definition of $\left[V_{i_{s}}\right]$. Hence $x \in \partial V_{i_{1}} \cap \cdots \cap \partial V_{i_{s-1}}$.

2.2. Lemma. Suppose that $f: X \rightarrow Y$ is a function, and $\mathcal{V}$ is a finite open cover of $X$. Then for every point $y \in Y$ we have

$$
\operatorname{ord}_{y} f([\mathcal{V}]) \leq \operatorname{ord}_{y} f(\partial \mathcal{V})+\left|f^{-1}(y)\right| \text {. }
$$

Proof. Let $\mathcal{V}=\left(V_{i}\right)_{i=1}^{m}$ and $T=\left\{i: y \in f\left(\left[V_{i}\right]\right)\right.$. To each index $i \in T$ we assign a point $\varphi(i) \in f^{-1}(y) \cap\left[V_{i}\right]$. We have

$$
\operatorname{ord}_{y} f([\mathcal{V}])=|T|=\sum_{x \in f^{-1}(y)}\left|\varphi^{-1}(x)\right| .
$$

For every $x \in f^{-1}(y)$, we can write $\varphi^{-1}(x)=\left\{i_{1}^{x}<\cdots<i_{\left|\varphi^{-1}(x)\right|}^{x}\right\}$ whenever this point-inverse is non-empty. 
According to the inclusion (1), we have

$$
y \in \bigcap_{\substack{x \in f^{-1}(y) \\\left|\varphi^{-1}(x)\right|>1}} f\left(\partial V_{i_{1}^{x}}\right) \cap \cdots \cap f\left(\partial V_{i_{\left|\varphi^{-1}(x)\right|-1}^{x}}\right) .
$$

Then, since $\left\{i_{1}^{x}, \ldots, i_{\left|\varphi^{-1}(x)\right|}^{x}\right\} \cap\left\{i_{1}^{\widehat{x}}, \ldots, i_{\left|\varphi^{-1}(\widehat{x})\right|}^{\widehat{x}}\right\}=\emptyset$ for $x \neq \widehat{x}$, we obtain

$$
\sum_{x \in f^{-1}(y)}\left(\left|\varphi^{-1}(x)\right|-1\right) \leq \sum_{\substack{x \in f^{-1}(y) \\\left|\varphi^{-1}(x)\right|>1}}\left(\left|\varphi^{-1}(x)\right|-1\right) \leq \operatorname{ord}_{y} f(\partial \mathcal{V}) .
$$

This inequality together with (2) completes the proof.

The next statement is a direct consequence of Corollary 2.5 and Theorem 4.3 in [16], where the notion of the partition dimension pdim of a map is used in formulations and proofs.

2.3. Lemma (cf. Aarts, Fokkink and Vermeer [1, Lemma 11]; Bogaty [5, Lemma 2]). Suppose that $f: X \rightarrow Y$ is a closed map all of whose point-inverses are zero-dimensional. Let $Y_{j} \subset Y$, where $j \in \mathbb{N}$, be $F_{\sigma}$-sets such that $\operatorname{dim} Y_{j}=n_{j}$. Moreover, take subsets $K_{1} \subset U_{1}, \ldots, K_{m} \subset U_{m}$ of $X$, where all the $K_{i}$ are closed, and $U_{i}$ are open. Then there exist open subsets $V_{1}, \ldots, V_{m} \subset X$ such that $K_{i} \subset V_{i} \subset \bar{V}_{i} \subset U_{i}$ for each $i$, and $\operatorname{ord}\left(f\left(\partial V_{i}\right) \cap Y_{j}\right)_{i=1}^{m} \leq n_{j}$ for every $j$.

2.4. Lemma. Suppose that $f: X \rightarrow Y$ is a closed map, $k \geq 2$, and define $C_{k}(f, \varepsilon)=\left\{y \in Y:\right.$ if $1 \leq m<k$ and $\widehat{x}_{1}, \ldots, \widehat{x}_{m} \in X$ are distinct,

$$
\text { then } \left.f^{-1}(y) \not \subset \bigcup_{i=1}^{m} \mathrm{~B}\left(\widehat{x}_{i}, \varepsilon\right)\right\} \text {, }
$$

where B indicates a ball. Then:

(a) Each $C_{k}(f, \varepsilon)$ is a closed subset of $Y$, and $C_{k}(f)=\bigcup_{\varepsilon>0} C_{k}(f, \varepsilon)$.

(b) If $y \in C_{k}(f, \varepsilon)$, then there are $x_{1}, \ldots, x_{k} \in f^{-1}(y)$ such that $\varrho\left(x_{i}, x_{j}\right)$ $\geq \varepsilon$ for $i \neq j$ (where $\varrho$ is the metric on $X)$.

Proof. $C_{k}(f, \epsilon)$ is the intersection of the sets

$$
\left\{y \in Y: f^{-1}(y) \not \subset \bigcup_{i=1}^{m} \mathrm{~B}\left(\widehat{x}_{i}, \varepsilon\right)\right\}=f\left(X \backslash \bigcup_{i=1}^{m} \mathrm{~B}\left(\widehat{x}_{i}, \varepsilon\right)\right),
$$

which are closed by the closedness of $f$.

Proof of Theorem 2.1. The inequality " $\leq$ ". Let $f: X \rightarrow Y$ and $k=$ ord $f$. Take a finite open cover $\left(U_{i}\right)_{i=1}^{m}$ of $X$. Choose a closed shrinking $\left(K_{i}\right)_{i=1}^{m}$ of $\left(U_{i}\right)_{i=1}^{m}$. By Lemma 2.3, there are open sets $V_{i}, i=1, \ldots, m$, such that $K_{i} \subset V_{i} \subset \bar{V}_{i} \subset U_{i}$ for each $i$, and $\operatorname{ord}\left(f\left(\partial V_{i}\right) \cap C_{j}(f)\right)_{i=1}^{m} \leq \operatorname{dim} C_{j}(f)$ for $j=1, \ldots, k$. Consider the reduction $[\mathcal{V}]$ of the cover $\mathcal{V}=\left(V_{i}\right)_{i=1}^{m}$. If $y \in Y$ 
and $\left|f^{-1}(y)\right|=j$, then (by Lemma 2.2) we have $\operatorname{ord}_{y} f([\mathcal{V}]) \leq \operatorname{ord}_{y} f(\partial \mathcal{V})+$ $j \leq \operatorname{dim} C_{j}(f)+j$. Thus, ord $f([\mathcal{V}]) \leq \max \left\{\operatorname{dim} C_{j}(f)+j: j=1, \ldots, k\right\}$.

The inequality " $\geq$ ". Since $\operatorname{dim} f(X) \leq \operatorname{cdim} f$, it remains to check that $\operatorname{dim} C_{j}(f)+j-1 \leq \operatorname{cdim} f$ for $j=2, \ldots, k$. Fix $j$, and write $n=\operatorname{dim} C_{j}(f)$. The countable sum theorem (see Engelking [8, Theorem 7.2.1]) implies that $\operatorname{dim} C_{j}(f, \varepsilon)=n$ for some $\varepsilon>0$.

Now, we confine our attention to the sets $Y^{\prime}=C_{j}(f, \varepsilon), X^{\prime}=f^{-1}\left(C_{j}(f, \varepsilon)\right)$, and the restriction $f \mid X^{\prime}$. As ord $f=k$, for every $y \in Y^{\prime}$ there are pairwise disjoint open (in $X^{\prime}$ ) sets $V_{i}^{y}$, where $i=1, \ldots, k$, such that $\operatorname{diam} V_{i}^{y}<\varepsilon$ for each $i$, and $f^{-1}(y) \subset \bigcup_{i=1}^{k} V_{i}^{y}$. (Some of these $V_{i}^{y}$ may be empty.) Choose a closed neighbourhood $F^{y}$ of $y$ such that $f^{-1}\left(F^{y}\right) \subset \bigcup_{i=1}^{k} V_{i}^{y}$. By the paracompactness of $Y^{\prime}$, we can assume that the family $\left\{F^{y}: y \in Y^{\prime}\right\}$ is locally finite. Define $K_{i}^{y}=V_{i}^{y} \cap f^{-1}\left(F^{y}\right)$, which are closed sets. According to the locally finite sum theorem (see [8, Theorem 7.2.3]), we have $\operatorname{dim} f\left(K_{i_{0}}^{y_{0}}\right) \geq n$ for some $y_{0}$ and $i_{0}$. Hence cdim $f \mid K_{i_{0}}^{y_{0}} \geq n$, and there exists a finite open cover $\mathcal{U}$ of $K_{i_{0}}^{y_{0}}$ that has no finite closed refinement $\mathcal{F}$ with ord $f(\mathcal{F}) \leq n$.

The sets of the following three kinds:

- $U \cup\left(V_{i_{0}}^{y_{0}} \backslash K_{i_{0}}^{y_{0}}\right)$, where $U \in \mathcal{U}$,

- $V_{i}^{y_{0}}$, where $i \neq i_{0}$, and

- $X^{\prime} \backslash f^{-1}\left(F^{y_{0}}\right)$

form an open cover, say $\mathcal{V}$, of $X^{\prime}$. We claim that $\mathcal{V}$ has no finite closed refinement $\mathcal{G}$ such that ord $f(\mathcal{G}) \leq n+j-1$. Indeed, suppose that a finite closed cover $\mathcal{G}$ refines $\mathcal{V}$. Since the sets $G \cap K_{i_{0}}^{y_{0}}$, where $G \in \mathcal{G}$, form a refinement of $\mathcal{U}$, there is a point $y \in \bigcap_{s=1}^{n+1} f\left(G_{s} \cap K_{i_{0}}^{y_{0}}\right)$ for distinct $G_{s} \in \mathcal{G}$. Since $\mathcal{G}$ refines $\mathcal{V}$, we have $G_{s} \subset V_{i_{0}}^{y_{0}}$ for each $s$. By Lemma 2.4, there are points $x_{t} \in f^{-1}(y)$, where $t=1, \ldots, j$, such that $\varrho\left(x_{t}, x_{t^{\prime}}\right) \geq \varepsilon$ for $t \neq t^{\prime}$. At most one of these points may belong to each of the sets $V_{i}^{y_{0}}$. There is one in $V_{i_{0}}^{y_{0}}$, maybe. Hence, there are further distinct sets $G_{t+n+1} \in \mathcal{G}$, where $t=1, \ldots, j-1$, such that $x_{t} \in G_{t+n+1}$. Thus, ord $f(\mathcal{G}) \geq n+1+j-1=n+j$. The foregoing claim means that $n+j-1 \leq \operatorname{cdim} f \mid X^{\prime} \leq \operatorname{cdim} f$.

3. The second formula for cdim $f$ and main corollaries. The next formula is more suitable for applications than that of Theorem 2.1.

3.1. THEOREM. If $f$ is a closed map from a non-empty space and ord $f$ $<\infty$, then

$$
\operatorname{cdim} f=\max \left\{\operatorname{dim} E_{j}(f)+j-1: j=1, \ldots, \text { ord } f\right\} .
$$

To prove this we need the following two lemmata. 
3.2. Lemma (J. Suzuki [26]). If $f$ is an exactly $k$-to-one closed map from a space $X$, then $\operatorname{dim} X=\operatorname{dim} f(X)$.

3.3. Lemma (Keesling [13, Corollary III.6], K. Nagami [23, Theorem 24-4]; the separable case: Hurewicz [12]). If $f$ is a finite-to-one closed map from a space $X$, then

$$
\operatorname{dim} C_{k}(f) \leq \max \left\{\operatorname{dim} X, \operatorname{dim} C_{k+1}(f)+1\right\} \quad \text { for each } k
$$

Proof of Theorem 3.1. Write $k=\operatorname{ord} f$. According to Theorem 2.1, it suffices to prove that

$$
\begin{aligned}
\max \left\{\operatorname{dim} E_{j}(f)+j-1: j\right. & =1, \ldots, k\} \\
& =\max \left\{\operatorname{dim} C_{j}(f)+j-1: j=1, \ldots, k\right\} .
\end{aligned}
$$

The inequality " $\leq$ ". By the theorem on dimension-lowering maps (see [9, Theorem 4.3.4]), we have $\operatorname{dim} E_{j}(f) \leq \operatorname{dim} C_{j}(f)$.

The inequality " $\geq$ ". Write $M=\max \left\{\operatorname{dim} E_{j}(f)+j-1: j=1, \ldots, k\right\}$. By downward induction we shall show that $\operatorname{dim} C_{j}(f)+j-1 \leq M$ for each $j$. Indeed, it follows from Lemma 3.2 that $\operatorname{dim} C_{k}(f)+k-1=\operatorname{dim} E_{k}(f)+$ $k-1 \leq M$ (for $j=k$ ). Assume that $\operatorname{dim} C_{j+1}(f)+j \leq M$. By Lemma 3.3 applied to the restriction $f \mid E_{j}(f)$ we obtain

$$
\begin{aligned}
\operatorname{dim} C_{j}(f)+j-1 & \leq \max \left\{\operatorname{dim} E_{j}(f), \operatorname{dim} C_{j+1}(f)+1\right\}+j-1 \\
& =\max \left\{\operatorname{dim} E_{j}(f)+j-1, \operatorname{dim} C_{j+1}(f)+j\right\} \leq M
\end{aligned}
$$

Since always $\operatorname{dim} f(X) \leq \operatorname{cdim} f$, Theorem 3.1 implies that if $f$ is a closed map from a non-empty space $X$ and ord $f<\infty$, then $\operatorname{dim} f(X) \leq$ $\max \left\{\operatorname{dim} E_{j}(f)+j-1: j=1, \ldots\right.$, ord $\left.f\right\}$. This theorem on dimension-raising maps was proved by Zarelua [29, 30] in a more general setting.

The next statement is a common generalization of several theorems on dimension-raising maps: Hurewicz [12], Zarelua [29, Corollary 1], [30, Proposition 4.5], Keesling [13, Theorem III.2], Nagami [23, Theorem 24-5] (see also A. Lelek [18] for a survey concerning maps and dimension inequalities).

3.4. THEOREM. Suppose that $f$ is a finite-to-one closed map from a nonempty space $X$. If the function $y \mapsto\left|f^{-1}(y)\right|$ takes on finitely many values $m_{1}<\cdots<m_{k}$ on $f(X)$, then

$$
\operatorname{dim} f(X) \leq \max \left\{\operatorname{dim} E_{m_{j}}(f)+j-1: j=1, \ldots, k\right\}
$$

Proof. Note that $f(X)=C_{m_{1}}(f)$, and repeat the reasoning in the proof of Theorem 3.1 (the inequality " $\geq$ "). Apply Lemma 3.2 to the restriction $f \mid E_{m_{k}}(f)$ and Lemma 3.3 to $f \mid E_{m_{j}}(f)$.

Theorems 3.1 and 1.2 immediately yield 
3.5. Corollary. If $f$ is an at most $k$-to-one closed map from a nonempty $n$-dimensional space $X$, then:

(a) $\operatorname{cdim} f \leq n+k-1$.

(b) There exists a closed map $g$ from a zero-dimensional space onto $X$ such that the composition $f \circ g$ is at most $(n+k)$-to-one.

(c) $f$ is a composition of $n+k-1$ simple closed maps.

3.6. REMARK. (i) In view of Remark 6.2 in [16] we infer that for any countably many at most $k_{i}$-to-one closed maps $f_{i}$ from an $n$-dimensional space $X$ there exists a closed map $g$ from a zero-dimensional space onto $X$ such that each composition $f_{i} \circ g$ is at most $\left(n+k_{i}\right)$-to-one.

(ii) If either $n=0$, or $k=3$ and $n$ is odd, then the number $n+k-1$ in (c) cannot in general be replaced by a smaller one; see [15, p. 151] and [25, Theorem 2].

4. Maps from spaces with Hurewicz's property $(\alpha)$. Motivated by the work of Hurewicz [11, 12], we impose additional assumptions on the map under which the number $n+k-1$ in Corollary 3.5 can be diminished.

A map $f: X \stackrel{\text { onto }}{\longrightarrow} Y$ is called irreducible if for every proper closed subset $F \subset X$, we have $f(F) \neq Y$.

4.1. Lemma (cf. G. T. Whyburn [28, Theorem 2], I. A. Vaŭnšteŭn [27, p. 30, Corollary 2]). If $f$ is an irreducible closed map from a space $X$, then $E_{2}(f)$ is a first category $F_{\sigma}$-set in $X$.

Proof. In view of Lemma 2.4(a), it suffices to show that $f^{-1}\left(C_{2}(f, \varepsilon)\right)$ is nowhere dense for every $\varepsilon>0$. Take any $\widehat{x} \in X$ and any number $\delta<\varepsilon$. Since $f$ is irreducible, there is a point $x$ such that $f^{-1} f(x) \subset \mathrm{B}(\widehat{x}, \delta)$. Hence $x \in \mathrm{B}(\widehat{x}, \varepsilon) \backslash f^{-1}\left(C_{2}(f, \varepsilon)\right)$.

4.2. Corollary. Suppose that $f$ is an irreducible, at most $k$-to-one $(k \geq 2)$, and closed map from an $n$-dimensional space $X$ that satisfies Hurewicz's condition $(\alpha)$. Then $\operatorname{cdim} f \leq n+k-2$, and so the conclusions of Corollary 3.5 remain true with $n+k$ replaced by $n+k-1$.

Proof. Since $X$ has property $(\alpha)$, we obtain $\operatorname{dim} E_{2}(f) \leq n-1$ by Lemma 4.1 and the countable sum theorem for $\operatorname{dim}$ (see [8, Theorem 7.2.1]). Use Theorems 3.1 and 1.2 to complete the proof.

We cannot drop the assumption of irreducibility in Corollary 4.2, for any exactly $k$-to-one map $f$ from an $n$-dimensional space has cdim $f=n+k-1$.

5. Maps from $n$-ACh spaces. In this section we find $n$-dimensional spaces that are not domains of at most $k$-to-one $(k \geq 2)$ closed maps $f$ with $\operatorname{cdim} f=n+k-1$. 
R. D. Anderson and G. Choquet [2] constructed a continuum that contains no pair of homeomorphic distinct non-degenerate subcontinua; see also J. J. Andrews [3]. (All known examples of such continua are one-dimensional.) These continua are a prototype for the following classes of spaces:

We say that a space of dimension $\geq n$ is $n$-ACh if it contains no pair of disjoint homeomorphic closed subspaces of dimension $\geq n$. Spaces that are 1-ACh are called Anderson-Choquet (abbrev. ACh).

It follows from the countable sum theorem (see [8, Theorem 7.2.1]) that in an $n$-ACh space, every $F_{\sigma}$-subspace of dimension $\geq n$ is also $n$-ACh.

This example shows that for every $n \geq 1$, there exists an $n$-dimensional n-ACh compactum:

5.1. Example. Consider the $n$-dimensional cube $I^{n}$, where $n \geq 2$, and choose a sequence of pairwise disjoint sets $D_{m} \subset I^{n}$ such that $\left|D_{m}\right|=m$ for each $m, \lim _{m \rightarrow \infty} \operatorname{diam} D_{m}=0$, and $\bigcup_{m=1}^{\infty} D_{m}$ is dense in $I^{n}$. Consider the decomposition $\mathcal{D}$ of $I^{n}$ into all the sets $D_{m}$ and all remaining singletons in $I^{n}$. It is easily checked that this decomposition is upper semicontinuous. We prove that the quotient space $A_{n}=I^{n} / \mathcal{D}$ is an $n$-dimensional $n$-ACh space.

Write $q: I^{n} \rightarrow A_{n}$ for the natural quotient map. By Lemma 3.3 with $k=1$, we obtain $\operatorname{dim} A_{n} \leq n$. We claim that $\operatorname{dim} q^{-1}(G)=n$ iff $\operatorname{dim} G=n$, for every subset $G \subset A_{n}$. Indeed, if $\operatorname{dim} q^{-1}(G)<n$, then $\operatorname{dim} G<n$-apply Lemma 3.3 to the restriction $q \mid q^{-1}(G)$. If $\operatorname{dim} q^{-1}(G)=n$, the theorem on dimension-lowering maps yields $\operatorname{dim} G=n$. In consequence, $\operatorname{dim} A_{n}=n$.

In order to show that $A_{n}$ has property $(\alpha)$, take an $n$-dimensional set $G \subset A_{n}$. Then $\operatorname{dim} q^{-1}(G)=n$, int $q^{-1}(G) \neq \emptyset$, and hence $\overline{I^{n} \backslash q^{-1}(G)}$ is a proper subset of $I^{n}$. Since $q$ is an irreducible map, we have

$$
\begin{aligned}
\emptyset \neq A_{n} \backslash q\left[\overline{I^{n} \backslash q^{-1}(G)}\right] & =A_{n} \backslash \overline{q\left[\overline{I^{n} \backslash q^{-1}(G)}\right]} \\
& \subset A_{n} \backslash \overline{q\left[I^{n} \backslash q^{-1}(G)\right]}=A_{n} \backslash \overline{A_{n} \backslash G}=\operatorname{int} G .
\end{aligned}
$$

Therefore, every n-dimensional subset of $A_{n}$ has non-empty interior.

Now, suppose that $h: M \stackrel{\text { onto }}{\longrightarrow} N$ is a homeomorphism between disjoint $n$-dimensional sets $M, N \subset A_{n}$. Then $h$ maps $U=M \backslash\left[\partial M \cup h^{-1}(\partial N)\right]$ onto $V=N \backslash[h(\partial M) \cup \partial N]$. As $M$ and $N$ have non-empty interiors, $U$ and $V$ are non-empty open subsets of $A_{n}$. Any point $D_{m} \in U$ disconnects every small enough connected neighbourhood $W$ of $D_{m}$ into $m$ components of $W \backslash\left\{D_{m}\right\}$. A contradiction: $V$ does not contain such a point with the same $m$.

It is not difficult to construct an $n$-dimensional $n$-ACh continuum without property $(\alpha)$. This can be done by starting with the product $S \times I^{n-1}$ instead of $I^{n}$, where $S$ denotes the $\sin 1 / x$ curve. 
5.2. THEOREM. If $k \geq 2$, then there exists no exactly $k$-to-one closed map from an $n$-ACh space.

Proof. Suppose that $f: X \stackrel{\text { onto }}{\longrightarrow} Y$ is such a map. It follows from Lemma 2.4(a) and the countable sum theorem that $\operatorname{dim} f^{-1}\left(C_{k}(f, \varepsilon)\right) \geq n$ for some $\varepsilon>0$. Lemma 2.4(b) implies that for every $x \in f^{-1}\left(C_{k}(f, \varepsilon)\right)$, the map $f$ is one-to-one on $\mathrm{B}(x, \varepsilon / 2) \cap f^{-1}\left(C_{k}(f, \varepsilon)\right)$. The restriction $f \mid f^{-1}\left(C_{k}(f, \varepsilon)\right)$ is a covering map, being an exactly $k$-to-one, locally one-to-one and closed map; cf. Krzempek [17, Lemma 1].

There is an open cover $\left(U_{i}\right)_{i \in I}$ of $C_{k}(f, \varepsilon)$ such that each $f^{-1}\left(\bar{U}_{i}\right)$ is the union of pairwise disjoint closed subsets $G_{i}^{j} \subset X$, where $j=1, \ldots, k$, and each $f \mid G_{i}^{j}$ is a homeomorphism onto $\bar{U}_{i}$. By the paracompactness of $C_{k}(f, \varepsilon)$, we can assume that the cover $\left(U_{i}\right)_{i \in I}$ is locally finite. From the locally finite sum theorem (see [8, Theorem 7.2.3]), we infer that some $G_{i}^{j}$ has dimension $\geq n$. This is impossible since $X$ is $n$-ACh.

5.3. Corollary. Suppose that $f$ is an at most $k$-to-one $(k \geq 2)$ closed map from an $n$-dimensional $n$-ACh space $X$. Then cdim $f \leq n+k-2$, and so the conclusions of Corollary 3.5 remain true with $n+k$ replaced by $n+k-1$.

Proof. If $E_{k}(f)$ were $n$-dimensional, it would be $n$-ACh. Hence, Theorem 5.2 leads to $\operatorname{dim} E_{k}(f) \leq n-1$. Use Theorems 3.1 and 1.2.

Applying Theorems 5.2 and 3.4, we obtain the following new result of the Hurewicz type; cf. Hurewicz [11, 12], Keesling [13, Theorem III.7].

5.4. Corollary. Suppose that $f$ is a closed map from an $n$-dimensional $n$-ACh space $X$. If the function $y \mapsto\left|f^{-1}(y)\right|$ takes on $k$ distinct values on $f(X)$, where $k \geq 2$, then $\operatorname{dim} f(X) \leq n+k-2$.

Observe that an $n$-dimensional space $X$ is $n$-ACh iff every simple closed map from $X$ is $n$-dimensional. Indeed, the sufficiency follows from Corollary 5.3. On the other hand, if $X$ contains two disjoint closed copies of the same $n$-dimensional space, then the simple map that glues these copies together has covering dimension $n+1$.

6. Open problems and remarks. Comparing the bounds of $\operatorname{dim} f(X)$ or cdim $f$ (for a map $f$ from a space $X$ ) in Corollaries 3.5, 4.2, 5.3, 5.4 and in theorems on dimension-raising maps from [11-13, 21], we can ask about stronger inequalities:

6.1. Question. Does there exist an $n$-dimensional compact space $X$, $n \geq 2$, such that $\operatorname{dim} f(X) \leq n+k-3[$ or even $\operatorname{cdim} f \leq n+k-3]$ for every at most $k$-to-one map $f$ from $X, k \geq 3$ ? 
The next proposition shows that if we knew Anderson-Choquet spaces of dimensions greater than one, they would be appropriate examples to the foregoing question.

6.2. Proposition. Suppose $f$ is an at most $k$-to-one closed map from an $n$-dimensional Anderson-Choquet space X. Then $\operatorname{cdim} f \leq \max \{n, k-1\}$, and so the conclusions of Corollary 3.5 remain true with $n+k$ replaced by $\max \{n+1, k\}$.

Proof. We can assume that $k=\operatorname{ord} f$. We claim that $\operatorname{dim} E_{j}(f) \leq$ $\operatorname{dim} E_{j+1}(f)+1$ for each $j \geq 2$. Indeed, it follows from the enlargement theorem (see [9, Theorems 4.1.19 and 4.1.3]) that there exists a $G_{\delta}$-subset $H \subset X$ such that $E_{j+1}(f) \subset H, \operatorname{dim} H=\operatorname{dim} E_{j+1}(f)$, and $H=f^{-1} f(H)$. The restriction $f \mid E_{j}(f) \backslash H$ is exactly $j$-to-one, and $E_{j}(f) \backslash H$ is an $F_{\sigma}$-set. If it had positive dimension, it would be an ACh space, and this would contradict Theorem 5.2. Thus $\operatorname{dim}\left[E_{j}(f) \backslash H\right] \leq 0$. The claim follows from the decomposition theorem (see [9, Theorems 4.1.16 and 4.1.3]).

We have $\operatorname{dim} E_{k+1}(f)=-1$, and our claim implies that $\operatorname{dim} E_{j} \leq k-j$ for $j \geq 2$. The proposition is a consequence of Theorems 3.1 and 1.2.

6.3. Question. Do there exist Anderson-Choquet spaces of dimensions greater than one?

We shall state a negative result in this direction.

The next definition is much more restrictive than that of an AndersonChoquet space: By a Cook continuum we understand a continuum $K$ such that every map $f: L \rightarrow K$ from a subcontinuum $L \subset K$ is either a constant map or the identity $\operatorname{id}_{L}$. The first example of such a continuum $K$ was constructed by H. Cook [7] (for a detailed description see A. Pultr and V. Trnková [24, Appendix A]); it was one-dimensional and hereditarily indecomposable.

T. Maćkowiak [19] showed that there exists no Cook continuum of dimension greater than two, and asked whether all Cook continua are curves. Since every continuum of dimension greater than $n$ contains a hereditarily indecomposable continuum of dimension at least $n$ (R. H. Bing [4]), the following statement implies Maćkowiak's:

6.4. Proposition. There exists no hereditarily indecomposable Cook continuum of dimension greater than one.

Proof. We modify the argument given in [19, (4.1)]. Recall that a map is monotone if each of its point-inverses is a continuum. A map $f: X \rightarrow Y$ is weakly confluent if for every continuum $B \subset Y$ there is a continuum $A \subset X$ such that $f(A)=B$.

Let $K$ be any hereditarily indecomposable continuum $K$ with $\operatorname{dim} K \geq 2$. Take a proper subcontinuum $X \subset K$ with $\operatorname{dim} X \geq 2$. Since $X$ is hereditarily 
indecomposable, by a theorem of J. L. Kelley [14] (see also K. P. Hart, J. van Mill and R. Pol [10]) there is a monotone open map $f: X \rightarrow Y$ onto an infinite-dimensional continuum $Y$. By a theorem of S. Mazurkiewicz [20] (see also S. B. Nadler [22, Theorem 13.56]) there is a weakly confluent map $g$ : $Y \rightarrow I^{3}$ onto the cube $I^{3}$. Take any one-dimensional continuum $Z \subset K \backslash X$ and an embedding $i: Z \rightarrow I^{3}$.

As $g$ is weakly confluent, there is a continuum $A \subset Y$ with $g(A)=i(Z)$. As $f$ is monotone, $L=f^{-1}(A) \subset X$ is a continuum; cf. [22, Exercise 8.46]. The map $i^{-1} \circ g \circ f \mid L: L \stackrel{\text { onto }}{\longrightarrow} Z$ shows that $K$ is not a Cook continuum.

\section{References}

[1] J. M. Aarts, R. J. Fokkink and H. Vermeer, Variations on a theorem of Lusternik and Schnirelmann, Topology 35 (1996), 1051-1056.

[2] R. D. Anderson and G. Choquet, A plane continuum no two of whose nondegenerate subcontinua are homeomorphic: an application of inverse limits, Proc. Amer. Math. Soc. 10 (1959), 347-353.

[3] J. J. Andrews, A chainable continuum no two of whose nondegenerate subcontinua are homeomorphic, Proc. Amer. Math. Soc. 12 (1961), 333-334.

[4] R. H. Bing, Higher-dimensional hereditarily indecomposable continua, Trans. Amer. Math. Soc. 71 (1951), 267-273.

[5] S. A. Bogatyı̆, The Lyusternik-Shnirel'man theorem and $\beta f$, Fundam. Prikl. Mat. 4 (1998), 11-38 (in Russian).

[6] K. Borsuk and R. Molski, On a class of continuous mappings, Fund. Math. 45 (1957), 84-98.

[7] H. Cook, Continua which admit only the identity mapping onto non-degenerate subcontinua, Fund. Math. 60 (1967), 241-249.

[8] R. Engelking, General Topology, PWN, Warszawa, 1977.

[9] —, Theory of Dimensions, Finite and Infinite, Heldermann, Lemgo, 1995.

[10] K. H. Hart, J. van Mill and R. Pol, Remarks on hereditarily indecomposable continua, Topology Proc. 25 (2000), 179-206.

[11] W. Hurewicz, Ueber stetige Bilder von Punktmengen (Zweite Mitteilung), Proc. Akad. Amsterdam 30 (1927), 159-165.

[12] —, Über dimensionserhöhende stetige Abbildungen, J. Reine Angew. Math. 169 (1933), 71-78.

[13] J. E. Keesling, Mappings and dimension in general metric spaces, Pacific J. Math. 25 (1968), 277-288.

[14] J. L. Kelley, Hyperspaces of a continuum, Trans. Amer. Math. Soc. 52 (1942), 22-36.

[15] J. Krzempek, Compositions of simple maps, Fund. Math. 162 (1999), 149-162.

[16] - Another approach to dimension of maps, Bull. Polish Acad. Sci. Math. 50 (2002), 141-154.

[17] - Covering maps that are not compositions of covering maps of lesser order, Proc. Amer. Math. Soc. 130 (2002), 1867-1873.

[18] A. Lelek, Dimension inequalities for unions and mappings of separable metric spaces, Colloq. Math. 23 (1971), 69-91.

[19] T. Maćkowiak, Terminal continua and the homogeneity, Fund. Math. 127 (1987), $178-186$. 
[20] S. Mazurkiewicz, Sur l'existence des continus indécomposables, ibid. 25 (1935), 327328.

[21] K. Morita, A condition for the metrizability of topological spaces and for $n$-dimensionality, Sci. Rep. Tokyo Kyoiku Daigaku Sec. A 5 (1955), 33-36.

[22] S. B. Nadler, Continuum Theory. An Introduction, Dekker, New York, 1992.

[23] K. Nagami, Dimension Theory, Academic Press, New York, 1970.

[24] A. Pultr and V. Trnková, Combinatorial, Algebraic and Topological Representations of Groups, Semigroups and Categories, North-Holland, Amsterdam, 1980.

[25] K. Sieklucki, On superposition of simple mappings, Fund. Math. 48 (1960), 217-228.

[26] J. Suzuki, Note on a theorem for dimension, Proc. Japan Acad. 35 (1959), 201-202.

[27] I. A. Vaŭnšteŭn, On closed mappings, Moskov. Gos. Univ. Učenye Zapiski Matematika 155 (1952), 3-53 (in Russian).

[28] G. T. Whyburn, On irreducibility of transformations, Amer. J. Math. 61 (1939), 820-822.

[29] A. V. Zarelua, On finite-to-one mappings, Dokl. Akad. Nauk SSSR 172 (1967), 775-778 (in Russian); English transl.: Soviet Math. Dokl. 8 (1967), 172-175.

[30] - Finite-to-one mappings of topological spaces and of cohomology manifolds, Sibirsk. Mat. Ž. 10 (1969), 64-92 (in Russian); English transl.: Siberian Math. J. 10 (1969), 45-63.

Institute of Mathematics

Silesian University of Technology

Kaszubska 23

44-100 Gliwice, Poland

E-mail: krzem@zeus.polsl.gliwice.pl

Received 3 January 2002;

in revised form 5 May 2004 